\title{
ENCLISIS PRONOMINAL Y POSICIÓN DEL VERBO EN ESPAÑOL ANTIGUO
}

Javier Elvira

Universidad Autónoma de Madrid

0. El presente trabajo contiene una propuesta de análisis de la colocación de los pronombres átonos en español medieval que examina la relación posible entre la movilidad del verbo y la tendencia enclítica del pronombre. Consta de cuatro apartados, el primero de los cuales repasa rápidamente las principales interpretaciones defendidas sobre esta cuestión. La segunda sección proporciona una casuística mínima de factores sintácticos que favorecen la anteposición o posposición del pronombre en relación con el verbo (en sus formas personales). En tercer lugar se examinan algunos aspectos generales y particulares de la colocación del verbo. El cuarto y último apartado plantea una interpretación de los datos presentados.

1. La atención de los estudiosos a este tema viene de muy antiguo. Ya en el siglo pasado, F. Diez dedicó algunas páginas de su conocido manual ${ }^{1}$ a comentar los usos románicos del pronombre, observando la tendencia del pronombre átono a acercarse al verbo y lo que para él era una mayor movilidad del pronombre medieval, en comparación con los usos modernos. Años después, Tobler publicó una serie de notas ${ }^{2}$ en las que examinó con cierta minuciosidad la imposibilidad del pronombre átono del antiguo francés de romper pausa y su posición nece-

'Grammatik der romanischen Sprachen, Bonn, 1844, vol. III, pp. 431-437.

2 Las principales referencias de A. Tobler al pronombre francés aparecieron en la $Z R P h$, en los número II, 1878, pp. 555-556; X, 1886, p. 169, y XIII, 1889, pp. 186-191. 
sariamente pospuesta al verbo cuando éste ocupaba la posición inicial. Sus observaciones suscitaron el interés de otros romanistas, que se propusieron examinar el mismo problema en las etapas antiguas de otras lenguas romances ${ }^{3}$.

Con las observaciones de estos autores quedaron sentadas las bases para una investigación que habría de durar muchos años. Los primeros intentos de explicación fueron tímidos y enfocaron el tema, la mayoría de las veces, desde un punto de vista acentual o rítmico. Desde el punto de vista sintáctico, hicieron énfasis en la estrecha vinculación entre el pronombre y el verbo, lo que llevó a algunos a defender la existencia de un doble uso proclítico o enclítico del pronombre, según que éste fuera antepuesto o pospuesto al verbo ${ }^{4}$. La idea de que un pronombre átono pudiera apoyarse en una parte de la oración diferente del verbo no fue siempre adecuadamente contemplada, a veces contra toda evidencia.

En 1892, Wackernagel publicó un sorprendente trabajo ${ }^{5}$ en el que estableció una ley, que lleva su nombre, según la cual, en las lenguas indoeuropeas antiguas (incluido el latín) las palabras átonas y, en general, las de escasa consistencia fónica tenían tendencia a apotarse como enclíticos en el primer elemento acentuado de la frase. Esta ley, sin embargo, ha sido puesta en duda posteriormente en lo que se refiere al latín. J. Marozeau ${ }^{6}$ señala que esta tendencia se observa en los autores latinos que él estudia como una herencia indoeuropea con escasa vigencia y múltiples excepciones. Ese mismo año, sin embargo, Thurneysen publicó un trabajo ${ }^{\top}$ en el que defendió, apoyándose en la ley de Wackernagel, el carácter eminentemente enclítico del pronombre con relación al elemento inicial, rompiendo con la idea de que el pronombre debiera apoyarse necesariamente en el verbo, ya como enclítico, ya como proclítico.

Por otros caminos llegó Meyer-Lübke a defender el carácter enclí-

${ }^{3}$ Cf. H, Tiktin, «Zur Stellung der Tonlosen Pronomina und Verbalformen im Rumänischen», ZRPh, IX, 1885, pp. 590-596; A. Mussafia, «Una particolarità sintattica della lingua italiana dei primi secoli», Miscellanea de Filologia e Linguistica in Memoria di $N$. Caix e U. A. Canello, Florencia, 1886, pp. 255-261; E. Gessner: «Das spanische Personalpronomen», ZRPh, XVII, pp. 1-54.

4 La consideración del pronombre como elemento básicamente ligado al verbo, ya sea enclítico o poclítico de éste, es el presupuesto básico de la llamada ley de Tobler-Mussafia, que pretende predecir la posición relativa de pronombre y verbo en función de los elementos que anteceden al verbo; cf.: A. Schiaffini, «Sulla legge Tobler-Mussafia», Testi Fiorentini, Florencia, 1954, pp. 275-283.

5 «Über ein Gesetz der indogermanischen Wortstellung», Indogermanische Forschungen, I, 1892, pp. 406-436.

6 L'ordre des mots en latin, Paris, 1953, vol. II, p. 90

7 «Zur Stellung des Verbums im Altfrazösischen», ZRPh, XVI, 1892, pp. 289-307. 
tico del pronombre, en un trabajo ${ }^{8}$ que ha sido el punto de referencia directa $o$ indirecta de gran parte de las discusiones posteriores. MeyerLübke se centró en el portugués, que no había sido objeto de atención específica, pero no olvidó manejar datos de otras lenguas, especialmente del español. En este trabajo llamó la atención de usos gráficos del antiguo portugués en los que el pronombre era representado formando una sola unidad gráfica con la palabra anterior (eute lexey), hecho que a su juicio era interpretable como una segmentación errónea del discurso favorecida por la naturaleza enclítica del pronombre. También presentó ejemplos de interpolación de elementos entre el pronombre y el verbo (este mundo em que se ho homem deleita), a partir de los cuales argumentó en contra de la supuesta vinculación entre el pronombre y el verbo ${ }^{9}$.

Sus datos, pues, parecían confirmar que los primitivos romances seguían la antigua ley de Wackernagel, u otra tendencia parecida, a la hora de colocar los elementos átonos en la frase. Si los usos son hoy día diferentes, ello se explicaría, según Meyer-Lübke, porque las lenguas románicas han experimentado un paso paulatino de la enclisis primitiva a la proclisis moderna. Este cambio sería un capítulo de un proceso más amplio que afectó a todas las partículas átonas.

No nos vamos a detener en la narración de todos los enfoques posteriores del problema durante del siglo ${ }^{10}$ porque todos ellos reformulan o matizan en mayor o menor medida las opiniones anteriores. Señalemos sólo que, en lo que se refiere al español antiguo, los estudios de Menéndez Pidal y Staaf ${ }^{11}$ continúan la interpretación enclítica del pronombre. Estos autores, aunque advierten que pudo haber condiciones sintácticas que regularon la posición del pronombre (circunstancias que ya había señalado Gessner), las consideraron excepciones a la interpretación enclítica.

También es obligado hacer una mención a un trabajo relativamente reciente sobre el tema que nos ocupa. Nos referimos al conocido libro

" «Zur Stellung der tonlosen Objektspronomina», ZRPh, XXI, 1897, pp. 313-334.

${ }^{y}$ Meyer-Lübke, op. cit., p. 317.

$10 \mathrm{Cf}$, entre otros, L. Foulet, «L'accent tonique et l'ordre des mots: formes faibles du pronom personnel après le verbe», Romania, L, 1924, pp. 54-93; E. Lerch: «Proklise oder Enklise der Altfranzösischen Objektspronomina? (Monistische und pluralistische Sprachanschauung)», ZRPh, LX, 1940, pp. 417-501; J. Melander, «Enklise oder Proklise des tonlosen Objektspronomens im Altfranzösischen", Studia Neophilologica, VIII, 193536, pp. 45-60; mucho más reciente es el estudio generativista de $M$. L. Rivero, «Parameters in the typology of clitics in Romance», man. inéd., Univ. de Ottawa, 1983. Anterior es el trabajo de Ramsden que citamos en nota 12.

11 E. Staaf, Etude sur les pronoms abrégés en ancien espagnol, Uppsala, 1906; «Contribution à la syntaxe du pronom personnel dans le Poème du Cid», Romanische Forschungen, XXIII, 1907, pp. 621-635. R. Menéndez Pidal, Cantar de Mio Cid. Texto, gramática y vocabulario, Madrid, 5.“ ed., 1977, vol. I, pp. 400-412. 
de Ramsden ${ }^{12}$, que tiene el valor de reunir una cantidad ingente de materiales de varias lenguas románicas (muy especialmente de la española) y de proporcionar un análisis del problema en algunos textos latino-tardios. Ramsden mostró, por un lado, que la colocación del pronombre respecto del verbo era todavía en latín tardío mucho más libre que en las lenguas romances y notó una mayor tendencia a la anteposición del pronombre en las oraciones subordinadas, hecho que atribuyó al mayor arraigo en subordinadas de la ordenación latina, que coloca el verbo al final. Por otra parte, su clasificación de los datos permitió establecer una relación entre la posición del pronombre y la naturaleza gramatical del elemento que ocupaba la posición inicial. A pesar del carácter marcadamente sintáctico de esta ordenación, Ramsden se decidió a proporcionar una interpretación rítmico acentual, como las que habían hecho fortuna en años anteriores.

2. Llegado este punto, y antes de cualquier discusión posible, parece necesario que intentemos precisar, aunque sea a grandes rasgos, cuáles son las circunstancias que parecen determinar la posición del pronombre en español medieval. Para ello nos vamos a servir de los datos que ofrece Ramsden y de un rastreo personal efectuado en varios textos medievales en prosa. Estos textos son La Fazienda de Ultramar ${ }^{13}$, el Liber Regum ${ }^{14}$, la Historia Troyana ${ }^{15}$, y la Primera Crónica General (2. ${ }^{\mathrm{a}}$ parte) ${ }^{16}$. Aunque Ramsden examinó también el Liber $R e$ gum, nos hemos decidido a volver sobre él no sólo por el interés que presenta un texto en prosa tan antiguo, sino porque nos interesa observar también otros aspectos sintácticos, como el orden de las palabras, que no preocuparon a Ramsden.

Basándonos en los datos que estas fuentes directas e indirectas nos proporcionan, creemos estar en condiciones de afirmar que la movilidad del pronombre átono en relación con el verbo parece depender de tres tipos de situaciones sintácticas:

A) Hay, en primer lugar, un grupo de circunstancias o contextos sintácticos en los cuales se produce siempre la anteposición del pronombre

${ }^{12}$ H. Ramsden. Weak-Pronoun Position in the Early Romance Languages, Manchester, 1963.

${ }_{13}$ Ed. de M. Lazar, Salamanca, 1965.

14 Ed. de L. Cooper, Zaragoza, 1960. 403.

15 Ed. de R. Menéndez Pidal en Textos Medievales Españoles, Madrid, 1976, pp. 223.

is Ed. de R. Menéndez Pidal, Madrid, Gredos, 1977. 
átono al verbo (en énfasis en la palabra «siempre» tiene la limitación propia que adquiere en la sintaxis medieval, donde las posibles excepciones aisladas a una tendencia general tienen normalmente una significación reducida). Dentro de este primer grupo vamos a distinguir cuatro situaciones:

- Cuando la negación antecede al verbo (cosa que ocurre en todos los casos) el pronombre átono va antepuesto:

no lo soterraron con los reies (LR, 5: 9) Nol podio connocer a dixol (Faz.: 47) Non lo podio mas celar (Faz.: 62) E non se alegraron en toda aquella noche (HTroy.-268:9-10) El conde non se le encubrio (PCG, p. 393: 27-28) et non lo puede escusar el uno nin ell otro (PCG, p. 398: 12-13)

- Provocan igualmente anteposición del pronombre algunos adverbios que anteceden al verbo modificándolo directamente; esto ocurre especialmente con los de lugar aquí, alli, de tiempo aun y de modo assi:

\& alli lo matoron amos sos fillos (LR, 4: 28) Al tiempo que los godos passoron mar, estonz se mouie Mahomat de Meca (LR, 12: 21) assi lo fizo (Faz.: 129) Depues, assi vos enbiare (Faz.: 63) e ally la soterro (Faz.: 44) E asy se engañan las mesquinas non pensando en su fazienda (HTroy-351: 25-26) e asi lo desamaron todas (HTroy.-305: 20) E ally se mostro el syn falla por tan brauo e $\tan$ esquiuo (HTroy.-303: 34-35) Aun se siguen las razones de la estoria de los fechos del noble don Alffonso (PCG, p. 681: 24-26) Aqui se acaba el regnado deste rey don Alffonso (PCG, p. 389: 9-10)

- Hay anteposición del pronombre cuando al verbo antecede un participio o infinitivo de él:

E partirse ia el flum Jordan a suso e a yuso (Faz.: 97) e demas por que nos ymos mucho legando a la hueste, rrogar vos ya e pedir vos ya merced (HTroy.-353: 18-19) e amar vos he sienpre lealmente (HTroy.-375: 19) De como este rey don Ramiro regno 
dicho lo auemos en el capitulo de ante desde (PCG, p. 389: 2122) et uençudos los auedes (PCG, p. 404: 2-3)

- Podemos afirmar que en las oraciones subordinadas el pronombre antecede al verbo con una regularidad casi absoluta ${ }^{15}$ :

Est crebanto la sepient... que encara la tienen los fillos d'Israel (LR, 5: 2) que non descercasse la uilla tro a que la prisiessen (LR, 17: 25) e murie assi como l'auia dito Aristotus (LR, 9: 29) e los que non se acogieren a las casas morran del pedrisco (Faz.: 67) E sy algund pesar o alguna desonrra les auian fecha fasta ally, commençaronse a vengar dellos (HTroy.-252: 30-32) En tod esto, quando el Çid Roy Díaz sopo que assi se paraua pleyto, et que se perderie la villa, et que la aurie el sennor de Denia... (PCG, p. 561: 14-17) Et pues que se non pudo tener, dexosse caer sobrel ninno (PCG, p. 555: 18-19) Pero ouieron miedo que les tenien los moros celadas en el camino (PCG, p. 333: 12-14).

En estas condiciones, la movilidad del pronombre es tal que en algunas ocasiones la atracción hacia el comienzo del período le lleva a separarse del verbo y da lugar a construcciones que resultan violentas para el uso moderno, en las que se intercala un elemento entre ambos ${ }^{18}$; el elemento intercalado suele ser la negación, un sujeto nominal o pronominal o un infinitivo dependiente del verbo subordinado:

...que se podiese defender atanto commo se el defendio (HTroy.296: 25-27) E si me el ama yaquanto / no he por quel desamar (HTroy.-IX: 101-104) non vos pese de quequier que uos yo agora diga (HTroy.-354: 21-22) muy grand derecho faze de se loar del vuestro amor el que lo auer puede (HTroy.-353: 12-13) A la hora que se don Hector fue desarmar, pararonse bien dozientas donzellas (HTroy.-305: 17-18) querria que sopiesedes por que me non quiso besar la mano el rey (PCG, p. 519: 7-8) Et duro la fa-

17 Fue Gessner, op. cit., pág. 40, el primero que observó la casi uniforme anteposición del átono en subordinadas. Menéndez Pidal, Cantar..., p. 403, ha observado también el fenómeno, pero lo considera una excepción a la interpretación enclítica que propone.

18 Cf. Menéndez Pidal, Cid, pp. 409-411. 
zienda siete dias que se non podian uencer los unos a los otros (PCG 332: 48-50)

Son éstas las construcciones que llamaron la atención de MeyerLübke en el trabajo que hemos citado anteriormente y que él consideraba un resto de usos presumiblemente más antiguos y una prueba palpable del carácter enclítico del pronombre y testimonio en contra de la supuesta inseparabilidad entre pronombre y verbo. Lo curioso es que Meyer-Lübke no advirtió o no quiso comentar que los ejemplos que proponía, procedentes del Libro de Alexandre, eran de oraciones subordinadas ${ }^{19}$.

Ejemplos de intercalación son bien conocidos en muchos textos medievales, aparte de los mencionados en este trabajo, y en todos ellos concurre con muy pocas excepciones la circunstancia sintáctica indicada:

que, si Dios quiere que se faga assi commo me el dixo, que seria mucho mi pro (El Conde Lucanor, p. 85) ${ }^{20}$ bien conosco a Dios que me a fecho muchas merçedes, mas quel yo podria servir (ibíd., p. 93) e si lo por bien toviesedes, ternia que seria bien que.. (Libro del Cauallero Zifar, p. 87) ${ }^{21}$ e luego cras en la mañana, nos iremos do nos Dios guiare (ibid., p. 83)

B) Encontramos, en segundo lugar, otro tipo de situaciones o contextos sintácticos que provocan normalmente la posposición del pronombre. Vamos a distinguir aquí cinco situaciones posibles:

- En primer lugar, el caso bien conocido en que el verbo ocupa la posición inicial (solo o precedido de la conjunción et):

e firieronlo i de una saieta (LR, 17: 23) E priso lo el rei Nabuchodonosor (LR, 5: 29) E trabajauanse quanto podian de se vengar de

19 En las pp. 314-315 de su trabajo citado, Meyer-Lübke cita ejemplos como los siguientes (procedentes del Libro de Alexandre): quanto lo mas calaua, quando te non vieren, etc.

${ }^{20}$ Citamos por la ed. de J. M. Blecua en Castalia, Madrid, 1969.

21 Citamos por la edición de J. González Muela en Castalia, Madrid, 1982. 
los griegos (HTroy.-275: 24-25) Et dixieronle esos caualleros quel connoscien (PCG, p. 763: 4-5) et fueronse para Molina (PCG, p. 595: 5-6)

- En segundo lugar, cuando la frase comienza con la conjunción mas y, en general, con las partículas o locuciones con valor coordinante:

mas leuantose Paris muy priuado e fue dar muy grand ferida (HTroy.-299: 16) mas cuytaualos tan fuerte el sagitario de cada parte, que... (HTroy.-317: 17-18) E por ende acogierense los troyanos para la villa (HTroy.-321: 3-4) mas dexola despues porque sospecho della (PCG, p. 386: 8-9)

- Hay, en tercer lugar, ciertos adverbios no incluidos en el apartado mencionado anteriormente, como desí, demás, que, cuando anteceden al verbo, provocan siempre la posposición. Estos adverbios no califican directamente al verbo ni forman con él una unión semántica estable y parecen desempeñar una función ilativa o conjuntiva:

e desy conbatieronse luego Paris con Agamenon (HTroy.-299: 8-9) E desy atarongela (HTroy.-265: 9) et desi fuesse con ellos poral rey (PCG, p. 375: 1-2) Desi dioles treguas fasta tiempo taiado et desi tornose pera su tierra de Leon (PCG, p. 385: 1415) Demas digouos que si yo... (PCG, p. 397: 18, E de mays, amigos, direuos otra cosa (HTroy.-321: 33-34)

- Cuando hay una subordinada o frase adverbial antepuestas a la principal, si el verbo ocupa la posición inicial de ésta, el pronombre va pospuesto:

Despues que ovieron fablado tornos Josep a Pharaon e dexol (Faz.: 58) Quando movio Jacob por Seyr, bendixol so padre Ysaach (Faz.: 48) e mientre fue Esau al canpo, dixolo ella a Jacob (Faz.: 47) $E$ desque fue desçendido del cauallo, abraçolo su madre (HTroy.-268: 1) et al cabo daquellos $V$ annos cegaronle (PGC, p. 391: 26-27) Et como quier que de luego llamassen a 
aquella çipdad çipdad de gloria, mudol el el nombre (PCG p. 680: 18-20)

- Finalmente, si el objeto directo o indirecto se anteponen al verbo, el pronombre del mismo caso, cuando lo hay, suele ir pospuesto:

e los qui eran de XI annos en suso. leuolos todos catiuos a Roma por mostrar su uictoria (LR, 11: 7) Tot el mal que so padre desfizo en Iherusalem refizo lo el com de cabo (LR, 5: 6) E Abraam a el diol el diezmo (Faz.: 44) Et estas mugeres ouolas una en pos otra (PCG, p. 520: 29-30) Et al conde aduzienle bien acompannados caualleros (PCG, p. 375: 39-40)

C) Podemos hablar, en fin, de un tercer grupo de situaciones sintácticas que no parecen tener una repercusión clara respecto al orden del pronombre. No la tienen en nuestros textos ni en los que ha examinado Ramsden. Son muchos los elementos que podrían incluirse en este apartado (frases preposicionales antepuestas, sustantivos con valor adverbial -essa hora-, etc). Nos vamos a referir solamente al caso en que hay un sujeto antepuesto, que resultará relevante en la discusión ulterior. Nuestros textos documentan abundantemente ambas soluciones ${ }^{22}$, sin que del examen de los ejemplos encontrados pueda deducirse el mecanismo posible que regula la posición del pronombre. La anteposición es posible con sujetos pronominales o nominales de toda índole:

Aquest se façia tener por deus (LR, 10: 28-29) Yol engennare (Faz.: 129) Dios le dara en tu mano (Faz: 127) el Sennor Dios de vuestros parientes se me apparecio e dixo (Faz.: 63) He yo me muero (Faz.: 60) los griegos uos enbian pedir treguas de tres meses (HTroy.-331: 6-7) e las vnas le tomauan el escudo, e las otras el espada (Htroy.-305) et este don Alffonso la poblo et la enlleno de yent (PCG, p. 679: 41-43) ell trabaio cutiano los da usados a ello (PCG, p. 680: 50-51 tu lo dexiste por el tu propheta Ysayas (PCG, p. 400: 31-32)

22 Sin embargo, Ramsden, op. cit., p. 77, afirma que aafter the subject of the verb the weak pronoun object usually precedes the verb». Los textos que hemos manejado no manifiestan en modo alguno esta preferencia, como vamos a mostrar. 
La posposición proporciona una casuística similar:

e muitos d'ellos tornoronse a la fe de Christus (LR, 12: 20) e ella tornos a la casa (Faz.: 47) e Jacob vynos a Socoth (Faz.: 50) Ella echol en el lecho del uaron de Dios (Faz.: 132) e los de la hueste acogieronse a las tiendas (HTroy.-325: 6) e el otro resçebiolo muy de grado (HTroy.-319: 24) E el rrey Neptolomo e el rrey Archilogo fueronse conbater anbos vno por otro (HTroy.-299: 26-27) Et Abderrahmen enuioles un so alcayde (PCG, p. 384: 1-2) El puerco acogiose a una cueva (PCG, p. 393: 46)

De los hechos que acabamos de presentar y ordenar podemos inferir que la naturaleza gramatical de la palabra que ocupa la primera posición de la frase o, al menos, la posición anterior al verbo es en muchos casos decisiva para fijar la situación del pronombre con relación al verbo. Nos preguntamos entonces por qué no puede apoyarse el pronombre en un objeto directo inicial, por qué se apoya siempre en determinados adverbios de lugar y vacila en presencia de un sujeto antepuesto.

Algunos de estos fenómenos fueron ya observados por Gessner, quien, a propósito de la dificultad de que un pronombre se apoye en un sujeto antepuesto, comentó en un tono más intuitivo que técnico que en estas construcciones parecía haber un cierto tipo de corte sintáctico que impedía el avance del pronombre. Gessner intuyó que, en algún sentido, esta anteposición del objeto constituía una anticipación y que, desde un punto de vista sintáctico, la frase comenzaba con el verbo o se comportaba «como si» empezara con éste ${ }^{23}$.

A nuestro juicio, la investigación tradicional del pronombre átono no ha conseguido después de más de un siglo de discusiones dar una formulación más precisa a la intuición de Gessner. La idea de Ramsden de que el grado de vinculación entre el verbo y el elemento que le precede es el factor que favorece o impide la anteposicion ${ }^{24}$, sin ser necesariamente incorrecta, adolece de la misma vaguedad de la vieja frase de Gessner, porque no hace explícito el alcance y sentido del término "vinculación» y su papel en la sintaxis del verbo medieval.

El objetivo de este trabajo, en lo que sigue, es intentar hacer algo más explícito el sentido de esta vinculación. Sería pretencioso intentar resolver en un artículo una cuestión que no se ha aclarado después de

${ }^{23}$ Gessner, op. cil., pp. 38-39.

24 Ramsden, op. cit., pp. 185 y ss. 
muchos años de discusiones. Pero sí deseamos aportar los datos que hacen defendible la idea de que, siendo la colocación del pronombre átono un aspecto más del tema más amplio de la ordenación de las palabras en español, la investigación sobre aquel tema podrá beneficiarse de los resultados que produzca el estudio del segundo.

3. La tarea de analizar los principios ordenadores de la frase medieval española ha proporcionado ya resultados estimables $y$, sin duda, podrá seguir ofreciéndolos en el futuro. Para evitar multiplicar los objetivos de este trabajo, vamos a apoyarnos en lo ya sabido ${ }^{25}$ y aportaremos algunas observaciones relativas a aspectos concretos de la colocación del verbo que puedan resultarnos relevantes.

En primer lugar, podemos confirmar la conocida tendencia del espanol a situar el verbo en una relación de anterioridad aunque no necesariamente de contigüidad con respecto a su objeto directo $o$ indirecto $o$, como afirma Lapesa, a respetar el esquema regente-regido ${ }^{26}$. Esto ocurre con una frecuencia del $95,7 \%$ en el Liber Regum, $86,7 \%$ en la Historia Troyana, $80,4 \%$ en La fazienda de Ultramar y $87 \%$ en la Primera Crónica General ${ }^{27}$. Que la ordenación VO está suficientemente arraigada en nuestros textos es algo que confirma no sólo la estadística sino la aparición nada infrecuente de fenómenos sintácticos que evidencian que la alteración de esa ordenación relativa violenta en algún sentido la ordenación básica de la lengua. Sabido es, por ejemplo, que en la lengua moderna la anteposición de un objeto directo $o$ indirecto (con actualización definida) al verbo de que depende provoca inmediatamente la aparición de un elemento pronominal enclítico que reproduce la referencia del elemento antepuesto. De esta forma, si invertimos la ordenación VO en la frase siguiente:

Puedes recoger las entradas en mi despacho

25 Como introducción general es muy útil el trabajo de $M$. Ariza, «Contribución al estudio del orden de palabras en español», Anuario de Estudios Filológicos, I, pp. 3-34, Cáceres, 1978, con bibliografia. Más específico es el libro de D. M. Crabb, A Comparative Study of Word Order in Old Spanish and Old French prose Work, Washington, 1955.

26 Cf. R. Lapesa, Historia de la Lengua Española, Madrid, 9." ed., 1981, n. ${ }^{\circ} 58$. La preferencia medieval por el orden VO se evidencia igualmente en las estadísticas efectuadas por D. M. Crabb, op. cit.

27 Sobre un total de 463 ejemplos en La Fazienda (pp. 116-157), 115 en el Liber Regum (pp. 19-28), 887 en la Primera Crónica General (pp. 489-528) y 391 en la Historia Troyana (pp. 232-263). Hemos simplificado deliberadamente nuestra estadística computando exclusivamente los casos de objeto directo o indirecto sustantivos. 
obtendremos la estructura siguiente:

las entradas puedes recogerlas en mi despacho

Construcciones de este tipo han sido objeto de atención dentro de la gramática generativa, que habla, de forma muy significativa, de una regla de dislocación a la izquierda ${ }^{28}$ que desplaza un constituyente, en este caso un SN, hacia la izquierda del verbo, dejando una copia pronominal en el lugar del elemento desplazado. La formulación de esta regla, según los autores que la han efectuado, presupone que la ordenación básica, profunda o subyacente es OV y que la aplicación de la regla en cuestión altera o disloca lo que en otra terminología podríamos llamar una ordenación «no marcada» 29.

Pues bien, el fenómeno de la dislocación a la izquierda con la consiguiente copia pronominal es conocido en la lengua de nuestros textos. Además de los casos que hemos citado, con otro propósito, en un apartado anterior, podemos proporcionar los siguientes ${ }^{30}$ :

e dos fillos que auia, fizo los degollar delant el (LR, 6: 9) Est fillo priso lo un ric omne de la montanna (LR, 16: 5) Ad est rei Clodoueo baptizolo Sant Remigu (LR, 18: 5) e tot el bien que so padre fizo desfizo lo el (LR, 5: 6-7) E Abraam a el diol el diezmo de todo lo que aduzie (Faz.: 44) $A$ los castellanos plogoles mucho de lo que el conde dixiera (PGC, pp. 401-402: 48-1) A la tierra de Guadalquivir pusol fuego ell arçobispo con la hueste (PCG, p. 681: 5-7) Las Alcarias con las pennas domolas con pueblos (PGC, p. 679: 45-46) et el desamor que ell auie contra ell tornolo en los fijos (PCG, p. 379: 48-49)

La segunda observación respecto al orden de las palabras en nuestros textos alude a la no menos conocida tendencia del verbo de la

28 Cf. M. L. Rivero: «On left-Dislocation and Topicalization in Spanish», Linguistc Inquiry, XI, 2, 1980, pp. 363-393.

29 Con esta misma noción de ordenación no marcada trabaja D. Prieto en su libro Prosodie et Syntaxe. La position des clitiques en galicien-portugais, Groningue, 1986, p. 55, que porporciona una interesante visión de la colocación de pronombre átono gallego-portugues.

${ }_{30}$ La aparición de copia pronominal se ve bloqueada en los casos en que está presente otro pronombre átono de caso distinto. En estos mismos casos de dislocación se produce la anteposición del pronombre:

Esto le lexo el Criador por amor de David (LR, 3:13)

E a el se aplegaran todos los pueblos (Faz.: 59) 
frase principal por situarse en posición inicial absoluta, solo o precedido de un adverbio ${ }^{31}$. Esta misma tendencia ha sido observada también en etapas antiguas de otras lenguas románicas ${ }^{32}$. En los textos manejados esta preferencia se da con una frecuencia del $55,5 \%$ en el Liber $R e$ gum, 71,4\% en La Fazienda de Ultramar, $75,3 \%$ en la Historia Troyana y 66,7\% en la Primera Crónica General ${ }^{33}$.

Esta ordenación inicial del verbo o sus constituyentes repercute en la disposición de las restantes categorías sintácticas. En particular, la posición del sujeto resulta afectada por la presencia inicial de algún elemento que modifique directamente al verbo, dando lugar a lo que suele denominarse, de una forma un tanto equívoca, inversión del sujeto. Observaciones semejantes han sido hechas ya respecto al español moderno por S. Fernández Ramírez ${ }^{34}$. En efecto, la frase:

Juan vino ayer

puede reordenarse de forma que el adverbio ocupe la posición inicial, pero tal reajuste provoca inmediatamente la inversión del sujeto:

Ayer vino Juan

En nuestros textos se produce igualmente esta inversión. Ocurre, por ejemplo, cuando la primera posición está ocupada por un adverbio o expresión adverbial:

Estonz fo Siria en poder de los romanos (LR, 11: 3) D'alli en acha fo França en poder de christianos (LR, 18: 5-6) Aqui s'estallo

${ }^{31}$ Cf. R. Lapesa, op. cit., n. ${ }^{\circ} 36.6$

32 Respecto del portugués puede verse el libro de Maria da Piedade Canaes e Mariz de Padua, $A$ ordem das palavras no portugues arcaico, Coimbra, 1960, que afirma en p. 133 que el orden VSO es uno de los más frecuentes en portugués arcaico e incluso en portugués moderno. La misma tendencia al verbo inicial ha sido observada en el francés antiguo; cf. J. Herman, «Recherches sur l'ordre des mots dans les plus anciens textes français en prose», Acta Linguistica Academiae Scientiarum Hungaricae, IV, 1954, pp. 6994 y 351-382 (especialmente p. 93) donde se habla de una evolución del orden latino en la que, tras una etapa de verbo inicial, se produjo primero una anteposición del adverbio y después del sujeto.

33 Sobre un total de 683 casos en el Liber Regum (pp. 18-28), 965 en La Fazienda de Ultramar (pp. 111-142), 518 en la Historia Troyana (pp. 302-329) y 1927 en la Primera Cronica General (pp. 533-552)

${ }^{34}$ Gramática española. El verbo y la oración. (Volumen preparado por Ignacio Bosque), Madrid, 1986, p. 434. 
la generacion de Charles (LR, 18: 24) Entre tanto regno Alexandre en Grecia (LR, 7: 18) Ally es Val de Lacrimarum (Faz.: 44) Apres vino sofijo Senacherib (Faz.: 135) e alli verie onbre tantas lorigas de cuerpo tan fermosas (HTroy.-360: 17-18) et oy en dia paresce y la sepultura dellos (PCG, p. 332: 5-6) Agora cuenta la estoria como... (PCG, p. 683: 52-53)

un complemento verbal preposicional:

A iii mygeros de torrente Cison es monte Gelbol (Faz.: 111) A cabo de tres dias comendo Josue a los sacerdotes que... (Faz.: 97) Al sol ponient de Antiochya es la Lycha (Faz.: 119) A aquella Tarsis cuedo foyr Jonas (Faz.: 119) E a la postremera noche del plazo tenien todos sus armas açecaladas e aguzadas (HTroy-360: 5-7) et en aquella az sera sant Yague (PCG, p. 401: 24-25)

una subordinada antepuesta:

Quando fo perdido el rei Rodrigo, conquerieron moros toda la tierra de Portugal (LR, 13: 18) Quando murio Sarra en Ebron, conpro Abraan la cueva doblada (Faz.: 44) Desque fue partido aquel consejo, fue ver Eneas a Elena (HTroy.-308: 17) Et pues que San Millan ouo esto dicho al conde, salio el de Sant Pedro (PCG, p. 401: 31-32) Luego que fue muerto el rey don Pelayo, alçaron los altos omnes a Phaphila su fijo rey (PCG, p. 578: 15-16)

un predicado nominal antepuesto:

Benedicto sea Abraan de Dios el Alto (Faz.: 44) mas quando el fue despierto, passada era aquella serpiente (PCG, p. 402: 45-46) Et seguro so yo de nos que non seremos uençudos (PCG, p. 403: 16-17) Et tan grand fue alli la mortandad dell un cabo et dell otro que... (PCG, p. 404: 30-32)

Por otra parte, podemos constatar, desde un punto de vista estadístico, que en los casos en que hay un sujeto explícito se observan dife- 
rencias en la relación de frecuencias del orden SV y VS en principales y subordinadas. En las oraciones principales, el orden VS aparece con una frecuencia superior al $60 \%$ en todos los textos $(78,1 \%$ en el Liber Regum, 88,4\% en La Fazienda de Ultramar, 69,8\% en la Primera Crónica General y $61,7 \%$ en la Historia Troyana) ${ }^{35}$. Esta preferencia por el sujeto pospuesto disminuye o se invierte en las oraciones subordinadas (72,4 \% en La Fazienda de Ultramar, $71 \%$ en el Liber Regum, $41,7 \%$ en la Primera Crónica General y $33,1 \%$ en la Historia Troyana) ${ }^{36}$.

Por lo demás, observamos una ordenación de la frase subordinada en la que abundan desplazamientos e inversiones menos usuales en la principal. No son raros, por ejemplo, los casos en que el verbo aparece en posición final absoluta ${ }^{37}$ :

d'i adelant non ouo rei ni princeb de lor lignage en Iherusalem, que d'i adelant a los caldeos siruieron (LR, 7: 6-7) Non ris, ca miedo of (Faz.: 45) Que tu todo esto firme myentre lo demandes (Faz.: 43) Juro el rey que a otro anno tanta gent adurie que... (Faz.: 127) Ante que los griegos saliesen de Tenedon, el castiello que nos de suso dixiemos (HTroy.-229: 6-7) et fabla y como si la cosa fuesse en aquel tiempo en que la estoria componie (PCG, p. 653: 19-24) Et quando ellos este miraglo oyeron, cogieronse a Santa María de Regla (PCG, p. 645: 27-28) sennor, quantos omnes uos aqui uedes, pero que ninguno non uos los dize... (PCG, p. 519: 28-29)

Todos estos datos nos permiten, en fin, suponer que la ordenación inicial del verbo en español medieval (o, al menos, en la lengua de los textos consultados) no está todavía claramente arraigada y que, en cualquier caso, la posición del sujeto respecto del verbo viene determinada en muchos casos por la presencia en posición inicial de una serie de elementos sintácticos directamente vinculados al verbo.

35 Sobre un total de $396,1.036,839$ y 816 ejemplos. Han sido examinadas con este fin las páginas 252-265 de la Historia Troyana, 93-137 de La Fazienda, 591-624 de la Primera Crónica General y 24-36 del Liber Regum.

36 Sobre un total de 101 ejemplos del Liber Regum, 165 en La Fazienda, 451 en la Primera Crónica General y 1.006 en la Historia Troyana.

${ }_{37}$ Estas diferencias en la ordenación del verbo en frases principales y subordinadas se han detectado ya en latín vulgar; cf. P. Linde, «Die Stellung des Verbs in der lateinischen Prosa», Glotta, XII, 1923, pp. 151-178 (especialmente 154-158) y J. N. Adams, "A typological Approach to latin Word-Orderw, Indogermanische Forschungen, LXXXI, 1976, pp. 70-99. 
4. Los hechos presentados en las páginas anteriores pueden condensarse en dos afirmaciones básicas:

a) Se detectan diferencias en la ordenación del verbo en las oraciones principales y en las subordinadas.

b) Se observan también diferencias en la colocación del pronombre átono en principales y en subordinadas. Mientras que la colocación del pronombre en principales está en función de la presencia en posición inicial de determinados elementos sintácticos, la posición del pronombre respecto al verbo en subordinadas es siempre antepuesta. Es de notar, además, la posibilidad en subordinadas de separar verbo y pronombre, intercalando entre ambos otro elemento sintáctico distinto.

La pregunta que surge inmediatamente es si existe alguna relación posible entre ambos hechos. En lo que sigue, intentaremos argumentar nuestra respuesta afirmativa a dicha pregunta.

$\mathrm{Si}$ aceptamos la consideración fundamentalmente enclítica del pronombre, como defendió Meyer-Lübke y continuó Menéndez Pidal, podremos observar que el pronombre avanza cuanto le es posible hacia el primer elemento de la frase y se apoya en aquellas palabras que no violan los principios ordenadores que creemos haber detectado.

Se apoya, en primer lugar, en un verbo inicial, que busca también la posición inicial en las oraciones principales. Se apoya igualmente en aquellos elementos que están estrechamente ligados al constituyente verbal, la negación, los participios o infinitivos dependientes y aquellos adverbios que modifican directamente al verbo ( $y$ no en aquellos que tienen valor conjuntivo).

Por el contrario, el pronombre no se apoya en aquellos elementos iniciales que no están ordenados de acuerdo con algunos de los principios generales observados: no suele apoyarse de un objeto directo o indirecto antepuesto porque esta ordenación parece alterar el esquema básico VO, como se pone de manifiesto en las construcciones dislocadas con copia pronominal que hemos presentado.

Por lo que se refiere a la vacilación respecto a la posibilidad de apoyar un pronombre en un sujeto antepuesto, parece razonable suponer que dicha vacilación puede estar relacionada con el hecho de que la ordenación SV en las oraciones principales de nuestros textos no es todavía más que una tendencia que no alcanza el $50 \%$ en ninguno de ellos.

Por último, la posibilidad de separar pronombre y verbo en oraciones subordinadas puede ponerse también en relación con las tendencias ordenadoras de la frase. La bibliografía sobre los clíticos, como 
hoy se les llama, es abundantísima ${ }^{38}$ y suele haber acuerdo en considerar que existe una vinculación sintáctica muy grande entre el átono y el verbo. Los generativistas, por ejemplo, consideran que la secuencia clítico + verbo funciona como un único constituyente sintáctico, con lo cual se formaliza la antigua observación de Díez sobre la progresiva vinculación de ambos elementos.

Se podría suponer que el análisis moderno de los clíticos valdría en español medieval sólo para aquellos que aparecen en oraciones principales, puesto que la separación de pronombre y verbo en subordinadas no es nada infrecuente, lo cual nos llevaria a hablar de una doble sintaxis del constituyente verbal que sería difícilmente justificable.

Hemos planteado la idea de que los textos medievales manejados presentan una tendencia a situar el verbo y sus constituyentes al principio de la frase principal. Sospechamos también, siguiendo a MeyerLübke, que el pronombre átono tiene tendencia a situarse como enclítico del primer elemento acentuado de la frase. En las oraciones principales, por tanto, se cruzan dos tendencias, una de carácter sintáctico, por la que el verbo busca la posición inicial; otra de carácter acentual por la que el pronombre busca también la posición inicial y se encuentra allí con el verbo. En cambio, en las subordinadas sólo encontramos la segunda tendencia, que mueve el pronombre hacia el comienzo de la frase, mientras que el verbo conserva todavía una mayor movilidad y puede incluso encontrarse en posición final absoluta, como hemos mostrado.

Si ello es así, no necesitamos suponer que en oraciones principales hay una sintaxis del constituyente verbal diferente a la de las subordinadas, sino que la movilidad del verbo es diferente en uno y otro caso. Thurneysen ${ }^{39}$ hizo afirmaciones parecidas a propósito del antiguo francés. Este autor observó también la tendencia del verbo francés por acercarse a la posición inicial, pero no la puso en relación con las tendencias ordenadoras de la frase sino con un proceso de debilitación acentual que afectó, según él, al propio verbo. No está claro que se pueda hablar de semejante debilitación respecto al verbo español. Lo que sí parece claro es que, por una u otra razón, el verbo y el pronombre átono se encontraron antes en la oración principal que en la subordinada y que debió ser allí donde se originaría una gramaticalización del constituyente verbo-clítico que terminaría extendiéndose a la subordinada.

${ }^{38}$ Cf. entre otros, A. C. Quicoli, *Some issues on the Theory of Cliticsw, Linguistic Analysis, X, 3, 1982, pp. 203-273; D. M. Perlmutter, Deep and Surface Structure Constraints in Syntax, Nueva York, 1971; R. Kayne, French Syntax: The Transformational Cycle, Cambridge, Mass, 1981, pp. 81 y ss.

39 Op. cit., pp. 305 y ss. 